\title{
Salud mental en la población peruana durante la COVID-19
} Mental health in the Peruvian population during COVID-19

\author{
Paola Llamocuro-Mamani, Frida Medrano-Espinoza* y David Montealegre-Soto
}

Escuela de Medicina Humana, Universidad Privada San Juan Bautista, Lima, Perú

\begin{abstract}
Resumen
La salud mental en respuesta a la pandemia de COVID-19 es un tema importante. En este artículo se hace una comparación en la población peruana dando a conocer diversos estudios, agregando los factores asociados a su desarrollo.
\end{abstract}

Palabras clave: Salud mental. COVID-19. Perú.

\begin{abstract}
Mental health as a response to the COVID-19 pandemic is an important subject. This article makes a comparison with the Peruvian population, releasing many studies, adding the factors associated with its development.
\end{abstract}

Key words: Mental health. COVID-19. Peru.

Al editor:

Hemos leído con mucha apreciación el artículo publicado por Pérez-Cano, et al. ${ }^{1}$ titulado Ansiedad, depresión y estrés como respuesta a la pandemia de COVID-19, cuyas conclusiones muestran que en población mexicana, de un total de 613 participantes, más del $40 \%$ presentaron ansiedad, el $41.3 \%$ depresión y menos del $30 \%$ estrés. Consideramos este estudio muy importante, ya que la salud mental es un tema fundamental en el contexto actual. En nuestro país se encontró, en 1064 participantes, que el 88.3\% presentaron síntomas como preocupación, temor e impotencia, y el $56.4 \%$ tuvieron estrés debido al aislamiento social ${ }^{2}$, cifras mayores en comparación con las del citado estudio'. En otro estudio con 67 participantes, el $43.3 \%$ presentaron ansiedad y el $20.9 \%$ tuvieron ansiedad leve, el $13.4 \%$ moderada y el $9 \%$ grave durante el aislamiento social ${ }^{3}$.

De la misma forma, un documento técnico del Plan de Salud Mental del Perú menciona que se realizó una encuesta sobre la salud mental de la población en el transcurso de la pandemia de COVID-19 y toque de queda, y de los 58,349 encuestados, el $28.5 \%$ presentaron síntomas asociados a la depresión; de ellos, se presentó mayormente en los de 18-24 años, siendo un $30.8 \%$ mujeres y un $23.4 \%$ hombres. Estas cifras son menores que las obtenidas en el estudio de Pérez-Cano, et al. ${ }^{1}$, en el que un $41.3 \%$ de la población presentó síntomas asociados a la depresión. Asimismo, el Ministerio de Salud del Perú brinda un Plan Estratégico para prevenir, vigilar, controlar y reducir el impacto de la pandemia que afecta la salud mental de la población ${ }^{4}$.

\section{Correspondencia:}

*Frida Medrano-Espinoza

Avda. José Antonio Lavalle s/n

Ex Hacienda Villa, Chorrillos

Fecha de recepción: 26-11-2020

C.P. 15067, Lima 1507, Perú

E-mail: frida.medrano@upsjb.edu.pe
Cir Cir. 2021;89(3):416-417

Contents available at PubMed www.cirugiaycirujanos.com ajo la licencia CC BY-NC-ND (http://creativecommons.org/licenses/by-nc-nd/4.0/). 
Entre los factores asociados a estas manifestaciones se han reportado los problemas económicos para comprar alimentos y medicinas durante la pandemia, familiares con COVID-19 y el tiempo de aislamiento social ${ }^{2}$. El uso inadecuado de los medios de comunicación puede generar desinformación, estigmatización del personal de salud, pánico de compra y aumentar el riesgo de ansiedad; motivo por el cual las personas deberían ser más selectivas con las noticias de televisión y el uso de las redes sociales no oficiales'. Por último, las recomendaciones que nos da la Organización Mundial de la Salud incluyen mayor inversión de recursos económicos en el sector de la salud mental, pues de seguir el curso que lleva la pandemia aumentará la demanda de estos servicios ${ }^{5}$.

\section{Agradecimientos}

Agradecemos a nuestros padres, que siempre nos dieron su apoyo incondicional.

\section{Responsabilidades éticas}

Protección de personas y animales. Los autores declaran que para esta investigación no se han realizado experimentos en seres humanos ni en animales.
Confidencialidad de los datos. Los autores declaran que en este artículo no aparecen datos de pacientes.

Derecho a la privacidad y consentimiento informado. Los autores declaran que en este artículo no aparecen datos de pacientes.

\section{Conflicto de intereses}

Los autores declaran no tener conflictos de intereses.

\section{Bibliografía}

1. Pérez-Cano HJ, Moreno-Murguía MB, Morales-López O, Crow-Buchanan O, English JA, Lozano-Alcázar J, et al. Anxiety, depression, and stress in response to the coronavirus disease-19 pandemic. Cir Cir. 2020;88:562-8.

2. Cuenca Jaque CR, Osorio Tarrillo ML, Pastor Ramos JL, Peña Pasapera GP, Torres Vásquez LE. Economic and health aspects in times of quarantine for COVID 19 in the Peruvian population, year 2020. Rev Fac Med Hum. 2020;20:630-9.

3. Oblitas González A, Sempertegui Sánchez N. Ansiedad en tiempos de aislamiento social por COVID-19. Chota, Perú, 2020. Avances en Enfermería. 2020;38:11-21.

4. Ministerio de Salud del Perú. Plan de salud mental (en el contexto COVID-19). Documento Técnico. MINSA. Perú; 2020. p. 13. Disponible en: https://www.gob.pe/institucion/minsa/informes-publicaciones/656521-plan-de-salud-mental-en-el-contexto-de-covid-19

5. Organización Mundial de la Salud. Los servicios de salud mental se están viendo perturbados por la COVID-19 en la \mayoría de los países, según un estudio de la OMS. 2020. (Consultado el 21 de ocubre de 2020.) Disponible en: https://www.who.int/es/news/item/05-10-2020-covid-19-disrupting-mental-health-services-in-most-countries-who-survey 